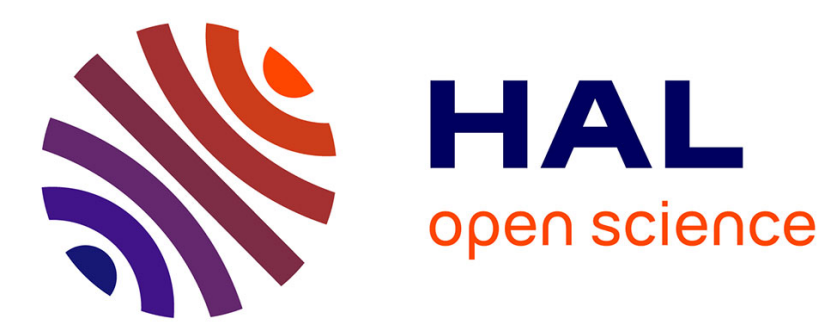

\title{
Application of Artificial Neural Network to Predict Static Loads on an Aircraft Rib
}

\author{
Ramin Amali, Samson Cooper, Siamak Noroozi
}

\section{To cite this version:}

Ramin Amali, Samson Cooper, Siamak Noroozi. Application of Artificial Neural Network to Predict Static Loads on an Aircraft Rib. 10th IFIP International Conference on Artificial Intelligence Applications and Innovations (AIAI), Sep 2014, Rhodes, Greece. pp.576-584, 10.1007/978-3-662-44654-6_57 . hal-01391362

\section{HAL Id: hal-01391362 \\ https://hal.inria.fr/hal-01391362}

Submitted on 3 Nov 2016

HAL is a multi-disciplinary open access archive for the deposit and dissemination of scientific research documents, whether they are published or not. The documents may come from teaching and research institutions in France or abroad, or from public or private research centers.
L'archive ouverte pluridisciplinaire HAL, est destinée au dépôt et à la diffusion de documents scientifiques de niveau recherche, publiés ou non, émanant des établissements d'enseignement et de recherche français ou étrangers, des laboratoires publics ou privés. 


\title{
Application of Artificial Neural Network to Predict Static Loads on an Aircraft Rib
}

\author{
Ramin Amali (ramin2.amali@uwe.ac.uk) \\ Samson Cooper (samson2.cooper@live.uwe.ac.uk) \\ University of the West of England, Bristol, UK \\ Siamak Noroozi (snoroozi@bournemouth.ac.uk) \\ Bournemouth University, UK
}

\begin{abstract}
Aircraft wing structures are subjected to different types of loads such as static and dynamic loads throughout their life span. A methodology was developed to predict the static load applied on a wing rib without load cells using Artificial Neural Network (ANN). In conjunction with the finite element modelling of the rib, a classic two layer feed-forward networks were created and trained on MATLAB using the backpropagation algorithm. The strain values obtained from the static loading experiment was used as the input data for the network training and the applied load was set as the output. The results obtained from the ANN showed that this method can be used to predict the static load applied on the wing rib to an accuracy of 92\%.

Keywords: Static load, Finite Element Analysis, Artificial Neural Network, Aircraft Rib, MATLAB

\subsection{INTRODUCTION}

Research into ANN and its application to structural engineering problems has grown significantly from increased interests over time. However, in the search for an optimum design, it is essential for engineers to evaluate the structural responses quickly at any design stage. Thus, the use of continuum models for the analysis of complex aircraft structures is an attractive idea in modern aerospace engineering especially at the conceptual and preliminary design stages [1]. Extensive research has been carried out using ANN to model operational loads experienced by a fixed-wing aircraft structure [2]. Flight loads on a fixed-wing aircraft can generally be separated into gust and manoeuvre
\end{abstract}


dominated loads, the majority of which tend to occur at frequencies of less than a few Hertz $(\mathrm{Hz})$ and in the case of a rotary-wing aircraft, the loading spectrum experienced by the airframe structure is significantly more complex. There have been a number of attempts in the last few decades at estimating these loads on the helicopter indirectly from flight state parameters or fixed points on the airframe with varying success [3].

In this work, several sets of data obtained from the static test conducted on a wing rib were analysed. This paper presents the implementation details of how accurate an artificial neural network has been used to predict the static load applied on the wing rib, the data sets are standard strain values recorded from the experimental test.

\subsection{EXPERIMENT}

The main aim of this research section was to measure the strain values which will be used as the inputs for the neural network training on MATLAB. The strain values are measured by subjecting the rib to a range of static loads using a powered hydraulic system.

\subsection{Experiment Design}

The rib is to be tested in two different positions due to the number of strain gauges and the location that they are attached on the rib. The testing equipment was set up as shown in Figure 1; the rib is supported in the frame with the aid of four steel rods bolted from the top surface of the shear tie to the top frame and another four steel rods connecting the bottom surface of the cap to the ground.

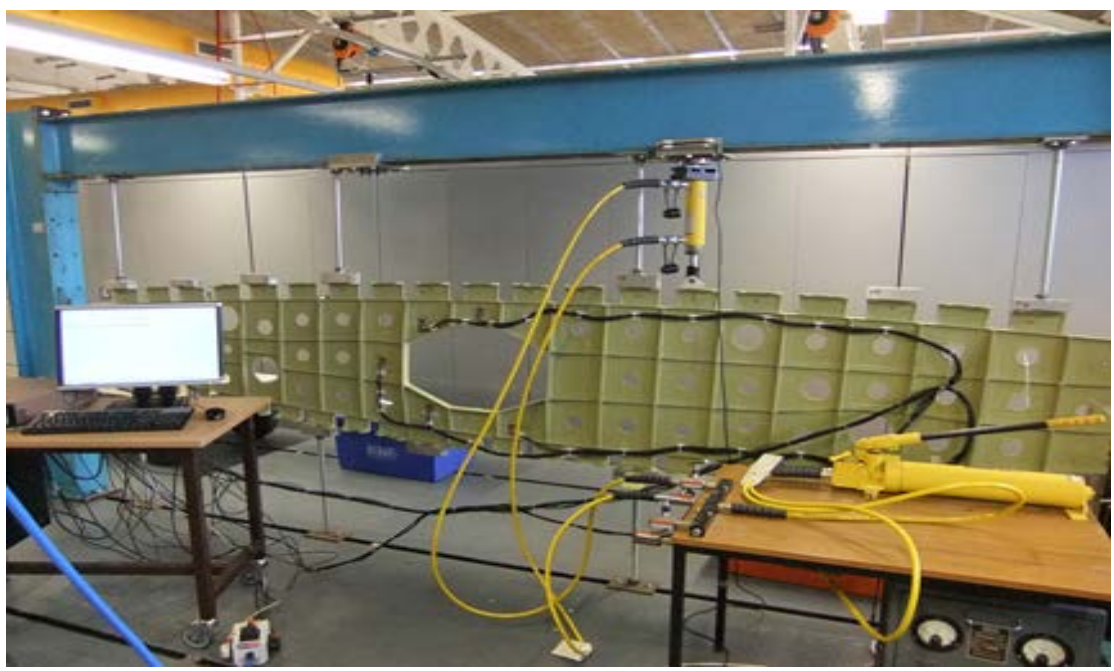

Figure 1: Static Experiment Set-up 
The fifteen strain gauges attached to the rib are channelled to the smart strain scanner which is in turn connected to a computer to run and record the strain values during the test process.

The cylinder is attached to the top part of the frame with a steel block bolted to the frame as shown in the experiment set-up; the cylinder is then connected to the manual hand pump with two high pressure hydraulic hoses as shown in Figure 1.

To conduct the test the manual hydraulic pump was used to apply a gradual load on the shear tie of the rib as shown above in Figure 1. The aim was to apply the specified load to the rib as quick as possible without physically damaging any part of the rib, while the smart strain scanner records the strain output of the rib due to the applied load over a time period. The software used for recording the strain output is precise enough to measure the applied load and strain to the nearest one percent.

However due to the fact that the test is a non-destructive test, the maximum load that could be potentially applied to the rib without causing any damage has been set to $3000 \mathrm{~N}$.

The strain data was gathered using the smart strain software, the overall test process was repeated three times on several locations on the rib where the cylinder comes in contact with the surface of the shear tie to get as many data and accurate results from the experiment.

\subsection{RESULTS}

The results obtained were analysed by plotting a graph to show the relationship between the force applied on the rib over a period of time and the output strain against time.

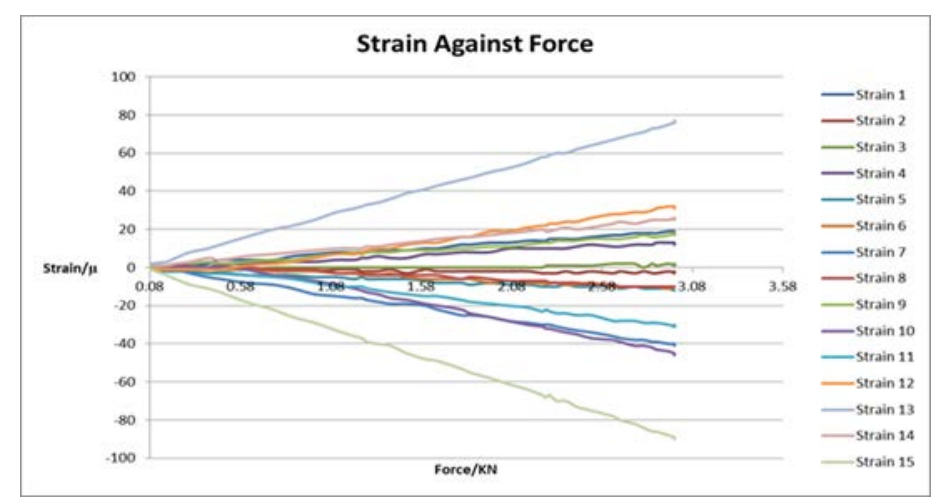

Figure 2: Strain against Force Graph-Experiment Result 
Figure 2 shows the relationship between the applied force and the measured strain from the experiment, it can be observed that the strain increases linearly as the force applied increases. Therefore it can be concluded that the relationship between the output and the input which would be used later in the neural network training are linearly related, this result also ascertain the assumption which was made before conducting the experiment.

\subsection{DATA ANALYSIS}

Before the network can be created it requires some data sets as inputs and targets obtained from the experiment, the data obtained from the experiment are in ordinary form of forces and strains. For the network to be trained it requires data from which it can learn the appropriate weight and biases that will help the network to produce the desired result. The data is obtained from the linear relationship between the force and strain graphs obtained from the experiment.
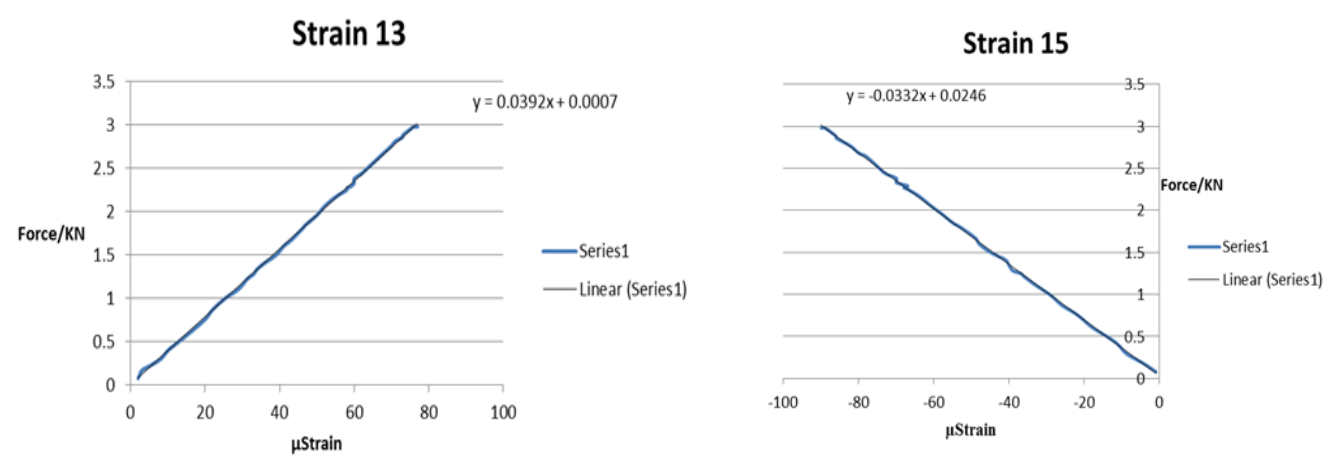

Figure 3: Force against Strain- Strain 13 and 15

To calculate the strain values which are used as the set of inputs for the network training, a line of best fit was plotted on each strain line in Figure 2 to obtain the linear relationship between the force and the strain. Figure 3 shows some examples of the line of best fit on the graph which also generates the equation that relates the parameters together.

The linear equation was attained for the fifteen strain lines present in each experiment which means that for each experiment there are fifteen linear equations relating the force and the strain which have two constant values.

In general the linear equation can be written in broad terms of the applied force and the strain as shown in the equation (1) 


$$
F=a_{n} \varepsilon_{n}+b_{n}, \quad n=1-15
$$

Where $\mathrm{F}$ is the force which indicates $\mathrm{y}$ in the equations in figure 3 , $\varepsilon_{n}$ is the strain indicating $\mathrm{x}$ on the graph, $\mathrm{a}$ and $\mathrm{b}$ are the intercept and gradient values in mathematical terms. By repeating the same step for the experimental test that was conducted, a number of equations are generated in generic terms as shown in equation (2)

$$
F_{1}=a_{n} \varepsilon_{n}+b_{n}, F_{2}=c_{n} \varepsilon_{n}+d_{n}
$$

Where $F_{1}$ and $F_{2}$ are the forces applied for two different experiments $\varepsilon_{n}$ is the corresponding strains and $\mathrm{n}$ represents strains 1-15 and

$a_{n} b_{n}, c_{n}$, and $d_{n}$, etc are constants for each corresponding strain line. Equation 2 represents a generic format of how the linear relationship between the force and the strain obtained from each experiment can be written.

By adding up $F_{1}$ and $F_{2}$ in the previous equation and rearranging to make $\varepsilon_{n}$ the subject, a set of strain values can be calculated from the equation which is used as the inputs for the training data. The generic form of the equation is written below:

$$
2 \varepsilon_{n}=\left(\frac{F_{1}-b_{n}}{a_{n}}\right)+\left(\frac{F_{2}-d_{n}}{c_{n}}\right)
$$

Finally, for the network to be trained accurately it requires a large number of data for the inputs and target. To achieve this, a set of random numbers ranging between zero and three were generated and then used to represent the targets which indicates the force in equation (3). Using the relationship constant obtained from each strain line graph of the experiment, the corresponding $\varepsilon_{n}$ values were calculated.

\subsection{NEURAL NETWORK IMPLEMENTATION}

This part of the investigation aims to use all the materials and results obtained from the previous section to create an ANN that will be able to predict the load applied to the rib under static conditions as shown in the experimental investigation. A number of neural networks were created for the different experiments carried out on the rib. The network was trained using the calculated strain values obtained through the strain relationship from the experiments as inputs and the corresponding applied load range as the training targets. The trained network was then used to predict the actual experimental load that was applied on the rib by using the measured strain values from the experiments as inputs, the results from the network were also compared with the exper- 
imental result to define how well the network was able to predict the applied load.

\subsection{TRAINING DATA}

The training data comprises of two sets which are the input sets and the targets sets, as previously mention the inputs are the calculated strains also referred to as $\varepsilon_{n}$ and the targets are the random numbers ranging from zero to three.

For the network training the inputs was set to a $100 \times 15$ matrix configuration and the corresponding set targets had a 100x2 matrix configuration, the $100 \times 2$ matrix represents the random forces ranging from zero to three and the $100 \times 15$ represents the corresponding calculated strain.

\subsection{NEURAL NETWORK CREATION}

The simulation and analysis of the network was executed on MATLAB using the neural network toolbox function. The first designed network is a two layer feed forward network with sigmoid functions, ten hidden neurons and two output functions feed forward network is mainly used for fitting multi-dimensional mapping problems when given consistent data. Figure 4 shows an illustration of the network configuration from MATLAB. This type of network is mainly used for input and output curve fitting which is required for this analysis. The network is trained with Levenberg-Marquardt back propagation algorithm.

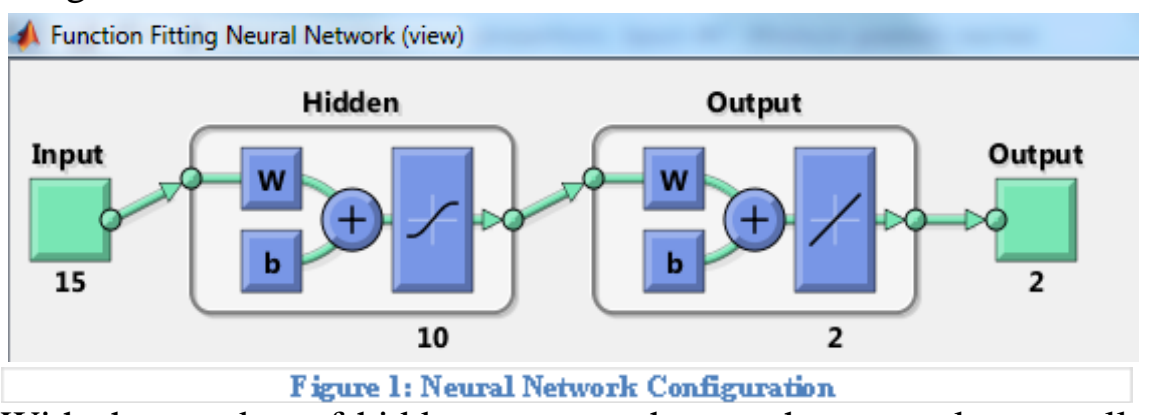

With the number of hidden neurons chosen, the network must allocate the data sets into three different parts which are training, validation and testing. The training data sets are presented to the network during training and the network is adjusted according to its error. The validation sets are used to measure network generalisation and to end training when generalisation stops improving. While the testing data sets which has no effect on the training is used to provide an independent measure 
of the network performance during and after training. For this neural network development, the default settings was selected on MATLAB which allocates $70 \%$ of the given data for training and $15 \%$ each for validation and testing.

\subsection{NETWORK VALIDATION}

The network created above uses a data set that its previously seen, therefore it is important to access the performance and capability of the trained network and how closely it would match other outputs values when a new set of inputs are introduced to the network. To validate the network a new set data calculated from the measured strains was set as the input in a $15 x 2$ matrix format to obtain a predicted load as the new output using the trained network. The output from the network is the predicted force or load which is then compared with the actual load that was applied to the rib during the experiment. Figure 5 shows an illustration of a bar chart comparing the predicted load obtained from the network with the experimental load.

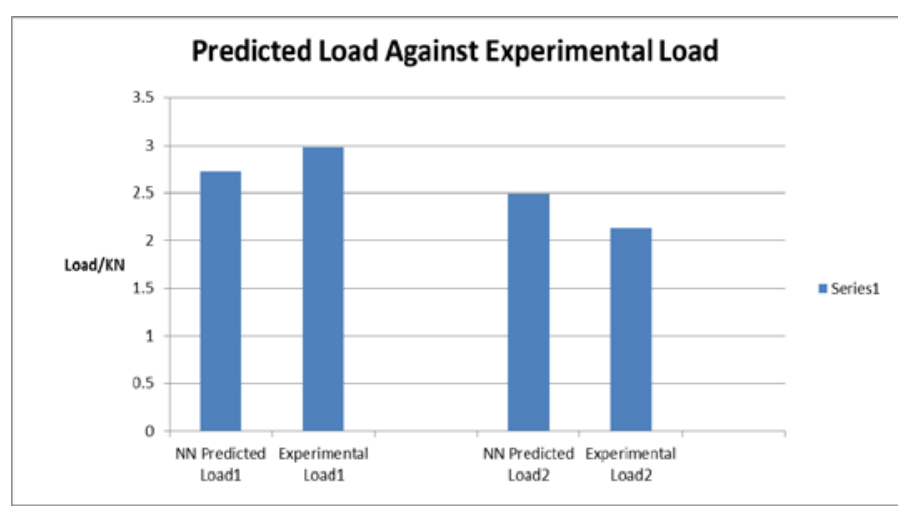

Figure 4: Neural Network Result comparison

The bar chart shows how well the network has been able to predict the load from the set of new strain data which was set as the new inputs. From the chart it can be observed that the error between the first predicted load and the experimental load is as low as 0.25 , which in percentage terms is approximately $8 \%$ and the second predicted load is 0.36 . The calculated error shows that the initial setting that was designed in the network before training the data and the selection of ten hidden neurons produces an appropriate result from the validation aspect of the network. To obtain a better accurate prediction an analysis 
was carried out on how the variation of the number of hidden neurons would affect the predicted load which is the output of a network.

\subsection{HIDDEN NEURON ANALYSIS}

For this analysis the inputs data was in a 300x15 matrix form and the target was in a 300x2 matrix form, the default settings was chosen on tool box for the data allocation where $70 \%$ of the data set was allocated to training and $15 \%$ each was allocated to both validation and testing.

The only value that is being varied in this analysis is the number of hidden neurons assigned to each network; however the number of hidden neurons for the analysis will range from two to fifteen which is a combination of the new set of inputs that would be used to validate each network created.

For all the networks trained for this analysis, a new set of data points was introduced to each network to obtain a new set of output which represents the predicted load, a graph was then plotted to show the relationship between the number of hidden neurons and the predicted load as shown in the graph plotted in figure 6. It is expected that an increase in the number of neurons in the hidden layer should initially improve the performance of the network, however there is a downside to increasing the hidden neurons. This causes the network to over fit the training data which will therefore reduce the effectiveness of the network when a new set of data is introduced to the network.

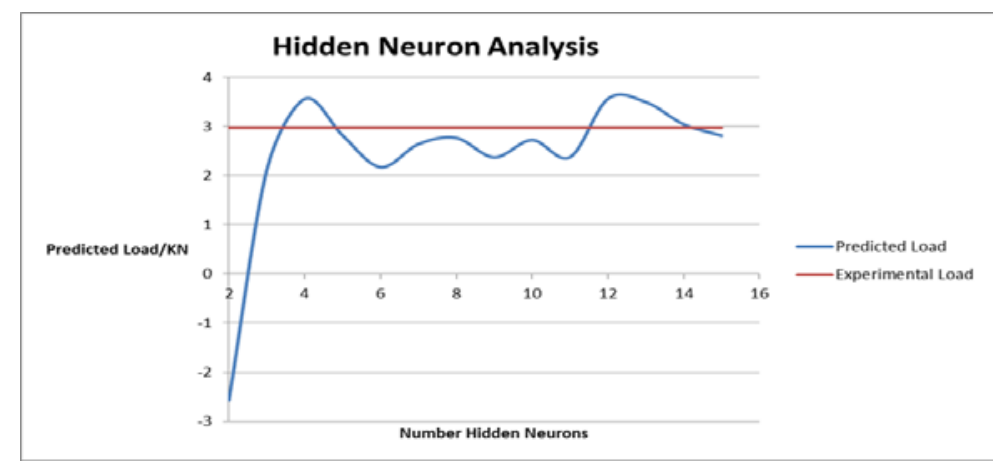

Figure 5: Hidden Neuron Analysis

Figure 6 shows the results obtained from the number of hidden neuron analysis, from the graph it can be observed that the network performance initially improves as the number of hidden neurons increases. The graph also shows that the network performs best in the range of 6 
to 10 hidden neurons as the predicted load obtained from those set of networks lie within the experiment threshold.

The results obtained from this analysis also indicates that there is a risk with increasing the number of hidden neurons where the network can begin to over fit the training data as shown in figure 6 . From eleven hidden neurons the predicted load starts to increases again, however to counter this effect it is possible to increase the number of data samples that is being introduced to the network for training.

\subsection{NEURAL NETWORK RESULT DISSCUSSION}

In conclusion the results for the entire neural network tests were positive as indicated from the range of several networks that was created. The results show that it is possible to predict or estimate the static load applied on the rib to an accuracy of $92 \%$.

However there is a requirement that must be met when training a network, this requirement is related to the number of hidden neurons used in training the samples and also the amount of data used to train and validate the network. To improve the accuracy of a neural network it is important to use as many data sets as possible, this will give the network more data samples to allocate for training, validation and testing which should lead to a better result.

Following the success of the prediction part of the networks, attempts were made to produce a method by which the networks would predict lower force values, this was achieved by changing the targets output and the targets of the network from the usually matrix format of [500x2] to a [500x1]. This new matrix will allow a new set of inputs in the matrix form of [15x1] to be introduced to the network to validate the performance of the network.

Also the new set of inputs values that was used for the training of this network has a range of strain values from correspond load applied between 0 and $1.1 \mathrm{KN}$, it is expected that the network should be able to predict the load as it different from the initial loads that have been predicted. The result obtained from this final validation shows that the network is able to predict any static load applied to the rib to an accuracy of $95 \%$ provided a consistent set of strain inputs are introduced to the network.

\section{CONCLUSION}

This paper presents the use of an ANN to predict the static load applied on a wing rib. It can be stated that this research has shown that it is possible to design a set of neural networks that will correctly predict 
or estimate the load applied on the rib provided a consistent set of strain inputs values are introduced to the network.

Although a large portion of the analysis has been carried out on the location of the rib where strain gauge rosettes have been attached, it is also possible to use the same technique to analyse other areas or the overall rib by attaching more strain gauges to the rib to capture accurate data when conducting similar static test.

The percentage error between the neural network prediction and the experiment can be classified as the error obtained from using some noisy data to train the network. This however does not affect the performance of the network or how well the network is able to predict other force values when a corresponding set of strain inputs are introduced to the network.

It should be stated that during the data analysis stage of the research, not all the fifteen strain gauges had a perfect linear relationship between the applied force and the measured strain, which could have also some error in the new data set that was used as inputs. Further improvement in the experimental procedure and more allocation of strain gauges to the rib will allow the measuring equipment to capture strain values more accurately which would lead to a better neural network creation and load prediction in the future.

\section{REFERENCES}

1. Liu, S. Tong, F., 2011. Fuzzy pattern recognition of impact acoustic signals for non-destructive. Sensors and Actuators: Physical Volume 167, pp.588-593.

2. Reed, S., Cole, D., "Development of a Parametric Aircraft Fatigue Monitoring System using Artificial Neural Networks", Proceedings of the 22nd Symposium of the International Committee on Aeronautical Fatigue, Lucerne, Switzerland, Mar 2003.

3. Polanco, F., "Estimation of Structural Component Loads in Helicopters: A Review of Current Methodologies", DSTO-TN0239, Dec 1999.

4. Mitchell, T. M. 1997. Machine Learning. New York: McGrawHill.85-100.

5. Bishop, C. M. 2006. Pattern recognition and machine learning. New York: Springer.226-290.

6. Mathworks, 2012. Neural Network Toolbox User Guide. s.l.:s.n 